\title{
INFLUÊNCIA DO DIAGNÓSTICO DE DIABETES MELLITUS GESTACIONAL NA QUALIDADE DE VIDA DA GESTANTE
}

\section{Luciana Ferreira Karsten}

Mestre em Saúde e Meio Ambiente pela Universidade da Região de Joinville (UNIVILLE); Coordenadora e Docente do Ensino Técnico de Enfermagem da Fundação Pró Rim (IPREPS) SC, Brasil.

E-mail: luleibis@yahoo.com.br

\section{Deisi Luciene de Souza}

Enfermeira pela Universidade do Vale do Itajaí (UNIVALI); Coordenadora e Docente do Ensino Técnico de Enfermagem da Fundação Pró Rim (IPREPS), SC, Brasil.

\section{Mariana Ramos Vieira}

Discente do $8^{\circ}$ semestre do curso de medicina da Universidade da Região de Joinville (UNIVILLE), SC, Brasil.

\section{Jean Carl Silva}

Doutor em Ciências Médicas pela Universidade Federal de São Paulo; Docente Programa de Pós-Graduação em Saúde e Meio Ambiente da Universidade da Região de Joinville (UNIVILLE), SC, Brasil.
RESUMO: O diagnóstico de Diabetes mellitus Gestacional proporciona alterações na rotina da gestante. Por conta disso, o objetivo deste trabalho foi comparar a qualidade de vida das gestantes antes e após o diagnóstico de Diabetes mellitus Gestacional e traçar o perfil das gestantes portadoras desse diagnóstico acompanhadas em um ambulatório de alto risco de maternidade pública. Trata-se de estudo observacional com 127 gestantes portadoras de Diabetes mellitus Gestacional (DMG), realizado de março a julho de 2014. Utilizou-se o questionário de Ferrans e Powers. Observou-se queda no grau de satisfação da QV no escore total (26,7 vs 25,3; DP: 2,0 vs 2,3; $p<0,01)$ e no IQV saúde/ funcionamento (26,8 vs 24,1; DP: 2,4 vs 3,4; $p<0,01)$ antes e após o diagnóstico. Nos outros itens avaliados não encontramos queda na QV (IQV família: 28,1 vs 28,1; DP: 2,4 vs 2,4; $p=0,986$; IQV socioeconômico: 26,8 vs 26,1; DP: 2,9 vs 3,0; $p=0,078$; IQV psicológico/ espiritual: 27,5 vs 27,0; DP: 2,9 vs 3,0; $p=0,196$ ). O DMG tem um impacto negativo na $\mathrm{QV}$ da gestante.

PALAVRAS-CHAVE: Diabetes mellitus; Gravidez; Qualidade de Vida.

\section{INFLUENCE OF DIAGNOSIS FOR DIABETES MELLITUS DURING PREGNANCY IN THE PREGNANT WOMAN'S LIFE QUALITY}

\begin{abstract}
Diagnosis of diabetes mellitus during pregnancy alters the pregnant woman's routine. Current analysis compares the life quality of pregnant women before and after diagnosis of diabetes mellitus during pregnancy and analyzes their profile in a high risk clinic of a government-run maternity. Current analysis, undertaken between March and July 2014, comprises 127 pregnant women with pregnancy diabetes mellitus (PDM), to whom the Ferrans \& Powers questionnaire was applied. A decrease in the satisfaction degree of LQ was reported in total score (26.7 vs 25.3; DP: $2.0 v$ s 2.3 ; $p<0.01)$ and in ILQ health/ functioning (26.8 vs 24.1; DP: $2.4 v s 3.4 ; p<0.01$ ) prior to and after diagnosis. No decrease in LQ (IQV family: $28.1 v$ s 28.1; DP: $2.4 v s$ 2.4; $p=0.986$; socio-economic ILQ: 26.8 vs 26.1; DP: 2.9 vs 3.0; $p=0.078$; psychological/spiritual ILQ: 27.5 vs 27.0; DP: 2.9 vs 3.0; $p=0.196$ ) was detected. PDM had a negative impact on the LQ of the pregnant woman.
\end{abstract}

KEY WORDS: Diabetes Mellitus; Pregnancy With Diabetes; Life Quality. 


\section{INTRODUÇÃO}

Com o passar dos anos a medicina sofreu inúmeras modificações e evoluções que conseguiram aprimorar a saúde da população proporcionando, dessa forma, a cura para diversas doenças que anteriormente eram letais. No entanto, outras várias patologias modificaram-se e constantemente continuam sendo estudadas e analisadas com o intuito de promover um convívio com o seu portador de forma branda, ou seja, para que esse indivíduo consiga obter um grau de qualidade de vida adequado (FERREIRA, 2013).

A sociedade passa por uma transformação importante onde um ambiente novo e composto de atividades individualista e consumista é visado como algo indispensável (OLIVEIRA; CARVALHO, 2014). Essa sociedade é seguramente uma sociedade de consumidores, atravessada pelos efeitos indesejáveis dos processos globalizadores, onde a urbanização, o estresse diário, os afazeres do cotidiano, duplas e triplas jornadas de trabalho, fast food, falta de exercícios físicos geram a um grande número de indivíduos doenças crônicas, que podem acarretar um prejuízo na sua qualidade de vida. Indiferente do gênero sexual das pessoas essas patologias podem prejudicar desde o convívio social como também a realização de tarefas simples de forma autônoma, ou, em outros casos, como na gestante, trazer consequências para o feto. Esse cenário está intimamente interligado quando se leva em consideração que qualidade de vida está relacionada a fatores ambientais, sociais, econômicos, fisiológicos e culturais (CARNEIRO; EUSÉBIO, 2011).

As mulheres, com o passar dos séculos, conquistaram espaço na sociedade mundial através de dedicação e desenvolvimento do seu conhecimento a trabalhos que até então eram realizados por homens. Entretanto, juntamente a partir dessa conquista a mulher também apresenta uma elevação no detrimento de sua qualidade de vida, pois realiza trabalhos extenuantes, possui novas preocupações, é mãe, desenvolve trabalhos domésticos, estuda, aprimora-se, enfim, diariamente são agregados à sua vida novos desafios e comprometimentos. Quando essa mulher inicia uma gestação ela precisa adaptar-se de forma psicológica e fisiológica à nova fase (SILVEIRA; FERREIRA, 2011).
A gestante fisiologicamente passa por alterações no seu organismo necessárias para manter a nova vida e, para isso, uma das modificações que ocorre é o aumento da taxa de glicose circulante, onde, a priori, é para ser metabolizada através da insulina que o pâncreas deveria estar produzindo. No entanto, em alguns casos isso não acontece ou, então, em outros episódios, a gestante possui previamente algum problema de metabolização de carboidratos acentuando-se na atual gestação, desenvolvendo, dessa maneira o Diabetes mellitus Gestacional (ABI-ABIB et al., 2014).

Todo esse quadro da fisiologia da gravidez, juntamente com o diagnóstico de uma doença crônica, se não possuir um comprometimento da gestante poderá concretizar a patologia de forma permanente. Sendo dessa maneira é indispensável que essa mulher conscientize-se do diagnóstico, bem como possa aderir ao tratamento para melhorar seu autocuidado (RAMOS, 2014). Frente à elevada taxa de pacientes diabéticos, especificamente as gestantes diabéticas e a correlação desse tema com a influência do meio ambiente sobre a qualidade de vida do portador, se faz necessário aprofundar esse tema.

Nesse sentido o estudo teve como objetivo comparar a qualidade de vida das gestantes antes e após o diagnóstico de Diabetes mellitus Gestacional e traçar o perfil das gestantes portadoras de Diabetes mellitus Gestacional acompanhadas em um ambulatório de alto risco de maternidade pública.

\section{METODOLOGIA}

Trata-se de estudo quantitativo, observacional, que avaliou a qualidade de vida antes e depois do diagnóstico de Diabetes mellitus Gestacional. A amostra constou de 127 gestantes entrevistadas no Ambulatório da Maternidade Darcy Vargas, na cidade de Joinville - Santa Catarina, no período de março a julho de 2014.

A Maternidade Darcy Vargas (MDV) de Joinville é uma instituição hospitalar gerenciada pelo Estado de Santa Catarina que tem como missão prestar assistência segura e humanizada à saúde integral da mulher, recém-nascidos e família, observando os princípios éticos. Auxilia na formação de profissionais nas áreas correlatas e promove o bem-estar no ambiente de trabalho, educação e saúde 
à comunidade e preservação do meio ambiente. A MDV atende no seu ambulatório gestantes com Pré-natal de Alto Risco, encaminhadas de Unidades Básicas de Saúde de Joinville e Região, além de consultórios particulares.

$\mathrm{O}$ projeto não apresentou conflito de interesse, pois os pesquisadores não possuíam relação com nenhuma indústria de medicamentos, equipamentos ou materiais utilizados na pesquisa.

Os critérios de inclusão para participar da pesquisa foram: gestantes maiores de 18 anos, com gestação única, com diagnóstico comprovado de Diabetes mellitus Gestacional conforme preconizado pela American Diabetes Association (STATEMENTS, 2012), acompanhadas no setor de Pré-Natal de Alto Risco da Maternidade Darcy Vargas, que compareceram nas consultas no período de março a julho de 2014, não tinham enfermidade associada e sem má formação fetal diagnosticada. Foram excluídas da amostra as pacientes que desistiram de participar do estudo durante a aplicação do questionário.

Para realizar a coleta de dados das gestantes, elas eram individualmente chamadas na sala de espera onde aguardavam para realizar consulta médica e controle de glicemia durante o pré-natal. As gestantes eram encaminhadas para uma sala reservada onde a pesquisadora realizava a explicação da pesquisa e lhe descrevia o Termo de Consentimento Livre Esclarecido (TCLE), documento que a entrevistada assinava e ficava com uma cópia assinada pela pesquisadora. Foram utilizados os dados maternos da carteira de gestante e o questionário de Qualidade de Vida de Ferrans e Powers. Em algumas coletas, devido ao preenchimento errôneo ou falta de dados na carteira da gestante, se fez necessário complementar essa coleta com a busca ativa através do prontuário das gestantes. Após essa assinatura, a pesquisadora iniciava o diálogo com a gestante para a coleta de dados, onde solicitava à entrevistada a sua carteira de gestante, para coletar os dados maternos (idade, idade gestacional no diagnóstico, número de gestações, diabetes gestacional anterior, se na gestação anterior foi parto cesárea ou se houve aborto, endereço, telefone, índice de massa corpórea (FAGUNDES et al., 2004), condições socioeconômicas familiares, tempo e tipo de tratamento (dieta, hipoglicemiante oral e/ou insulina).

O questionário sobre qualidade de vida de Ferrans e Powers utilizado - Quality of Life Index-Diabetes
Version III, foi devidamente validado e traduzido, sendo desenvolvido pelas enfermeiras (PhD) Carol Estwing Ferrans e Marjorie Powers da Universidade de Illinois, de Chicago (Estados Unidos), em 1984, para medir a qualidade de vida em termos de satisfação com a vida. Esse questionário é constituído por 34 questões de perguntas fechadas e com respostas que são enumeradas de 1 (sem importância) a 6 (muito importante), refletindo a satisfação com os aspectos da vida que são valorizados pelo indivíduo. $O$ valor da importância que o entrevistado refere é sobre inúmeros questionamentos gerais sobre a qualidade de vida. Essas 34 questões são agrupadas em cincos domínios pré-estabelecidos por seus desenvolvedores: índice geral de qualidade de vida, índice de qualidade de vida na saúde e funcionamento, índice de qualidade de vida familiar, índice de qualidade de vida socioeconômico e índice de qualidade de vida psicológico e espiritual.

No segundo momento da entrevista, a pesquisadora explicava qual a finalidade do questionário Ferrans e Powers, bem como ele era estruturado e quais as repostas que a gestante deveria fornecer (numeração e o significado de cada resposta). Era salientado à gestante que não havia questões com respostas corretas ou incorretas e que as respostas a cada questão eram fruto de sua interpretação, portanto a gestante deveria pensar por alguns segundos antes de responder. Para que a gestante pudesse responder o questionário era preciso que a mesma lembrasse como eram seus hábitos antes do diagnóstico de Diabetes mellitus Gestacional e após esse diagnóstico. Sendo assim, as 34 perguntas foram aplicadas duas vezes na mesma entrevista, pois o questionário é dividido em duas partes, totalizando 68 respostas. A primeira parte questionava à gestante o quanto ela estava satisfeita com sua vida; na segunda parte buscou apanhar quais os itens que ela considerava mais importantes para sua vida.

Essas 68 respostas foram dispostas em uma planilha do programa Microsoft Excel ${ }^{\circledR}$, pré-estabelecida por seus desenvolvedores na sua organização e dados calculados, que fornecia um escore em cada um dos cinco domínios, para posteriormente serem analisados conforme avaliação dos dados estatísticos da pesquisadora.

$\mathrm{Na}$ determinação estabelecida pelos desenvolvedores, após o lançamento das respostas na planilha do programa Microsoft Excelß, cada domínio gerou um 
escore individual que apresenta pontuação máxima em qualidade de vida no valor de 30, abaixo desse valor os desenvolvedores sugerem que a qualidade de vida seja avaliada conforme a perspectiva de cada estudo, ou seja, não há uma determinação específica mencionando valores para sugerir menor ou uma média qualidade de vida do pesquisado. Dessa forma, o escore que foi resgatado com o estudo é comparado com valores de outras pesquisas.

Após a coleta, todos os dados foram tabulados e analisados com auxílio do programa Microsoft Excel ${ }^{\circledR}$ e posteriormente feita análise estatística pelo programa SPSS versão 21. Inicialmente todas as variáveis foram analisadas descritivamente. Para as variáveis quantitativas esta análise foi feita através do cálculo de médias e desvios-padrão. Para as variáveis qualitativas calcularam-se frequências absolutas e relativas. Para a análise da hipótese de igualdade entre a média dos dois grupos foi utilizado o teste $t$ de Student, quando a suposição de normalidade foi rejeitada utilizou-se o teste não-paramétrico de Mann-Whitney. O nível de significância utilizado para os testes foi de $5 \%$.

A pesquisa respeitou o sigilo da identidade das pesquisadas, que é legalmente amparado nas normas da Resolução do Conselho Nacional de Saúde 466/12 (CNS, 2013) e utilizou o Termo de Consentimento Livre Esclarecido (TCLE), com a autorização das gestantes a participarem da pesquisa. As entrevistadas tiveram o direito de não participarem do estudo sem nenhum tipo de penalidade. A pesquisa passou pela autorização do Comitê de Ética em Pesquisa em Seres Humanos da Universidade da Região de Joinville com número de protocolo 492.552/2013. E, após essa autorização, buscou-se a autorização no Departamento de Pesquisa da Maternidade Darcy Vargas.

\section{RESULTADOS}

Foram entrevistadas 127 gestantes com diabetes gestacional, sem outras comorbidades, maiores de 18 anos, sem gemelares ou má formação congênita, não houve exclusões.

Na Tabela 1 pode-se observar que a média de idade das gestantes entrevistadas é de 29,8 anos, bem como a maioria estava passando pela nova gestação, pos- suindo IMC de $31,8 \mathrm{~kg} / \mathrm{m}^{2}$. No dia da entrevista estava com 32,5 semanas de gestação em média e a maioria perfazia de uma renda familiar de 2 a 3 salários mínimos.

Tabela 1. Características maternas de 127 pacientes com $\mathrm{DMG}^{*}$ acompanhadas no ambulatório da Maternidade Darcy Vargas, Joinville (SC), 2014

\begin{tabular}{|c|c|c|}
\hline Variável & Média (DP) ${ }^{\dagger}$ & Frequência (\%) \\
\hline Idade & $29,8(7,0)$ & \\
\hline Gestações totais* $^{*}$ & $2,7(1,6)$ & \\
\hline $\mathrm{IMC}^{* * *}$ & $31,8(5,7)$ & \\
\hline IG**** coleta (semanas) & $32,5(4,8)$ & \\
\hline \multicolumn{3}{|c|}{ Renda familiar (salários mínimos) } \\
\hline Menos de 1 & & $5(3,9)$ \\
\hline 1 & & $13(10,2)$ \\
\hline 2 a 3 & & $49(38,6)$ \\
\hline 3 a 5 & & $45(35,4)$ \\
\hline Mais de 5 & & $15(11,8)$ \\
\hline
\end{tabular}

${ }_{\dagger}^{\dagger}$ Média e desvio padrão; ${ }^{\ddagger}$ Frequência e percentil; ${ }^{*}$ Diabetes $m e l$ litus Gestacional; "* Número de gestações prévias; ${ }^{* * *}$ Índice de massa corpórea; ${ }^{* * * *}$ Idade gestacional.

Sobre as características maternas das entrevistas relacionadas ao Diabetes, na Tabela 2 pode-se notar que a idade gestacional no diagnóstico é, em média, de 24,6 semanas. $O$ tempo de tratamento que essas gestantes possuíam no dia da coleta foi em média de 61,1 dias e 55,9\% das entrevistadas utilizavam a dieta como meio exclusivo para o controle glicêmico. Das 127 entrevistadas, 24 estavam passando por uma nova ocorrência de Diabetes mellitus Gestacional.

Tabela 2. Características do tratamento de 127 pacientes com DMG* acompanhadas no ambulatório da Maternidade Darcy Vargas, Joinville (SC), 2014

\begin{tabular}{lcc}
\hline \multicolumn{1}{c}{ Variável } & Média (DP) ${ }^{\dagger}$ & $\begin{array}{c}\text { Frequência } \\
(\%)^{\ddagger}\end{array}$ \\
\hline $\begin{array}{l}\text { IG* diagnóstico DMG } \\
\text { (semanas) }\end{array}$ & $24,6(8,5)$ & \\
Tempo de tratamento (dia) & $61,1(64,7)$ & \\
\hline Tipo de Tratamento & $11(8,7)$ \\
\hline Sem tratamento & $71(55,9)$ \\
$\quad$ Dieta & $28(22,0)$ \\
$\quad \begin{array}{l}\text { Dieta e hipoglicemiante oral } \\
\text { Dieta, higoglicemiante oral e insulina de trata- }\end{array}$ & $10(7,9)$ \\
mentos & $7(5,5)$ \\
$\quad \begin{array}{l}\text { Dieta e insulina } \\
\text { Presença de DMG prévia }\end{array}$ & $24(18,9)$ \\
\hline
\end{tabular}

${ }_{\dagger}^{\dagger}$ Média e desvio padrão; ${ }^{\ddagger}$ Frequência e percentil; ${ }^{*}$ Diabetes mellitus Gestacional; ${ }^{* *}$ Idade gestacional. 
A Tabela 3 demonstra as satisfações e a importância da qualidade de vida para a casuística utilizando o instrumento de Ferrans e Powers - Quality of Life Index - Diabetes Version III. Observa-se nessa tabela que houve alteração na percepção da importância da qualidade de vida da gestante no domínio IQV geral e saúde e funcionamento $\operatorname{com} p<0,01$ e uma queda no índice geral de qualidade de vida e saúde e funcionamento.

Tabela 3. Média e Desvio Padrão dos escores do Índice de Qualidade de Vida em gestantes com DMG* acompanhadas no ambulatório da Maternidade Darcy Vargas, Joinville (SC), 2014

\begin{tabular}{|c|c|c|c|c|}
\hline \multirow{2}{*}{\multicolumn{2}{|c|}{ Domínios }} & \multirow{2}{*}{$\begin{array}{c}\text { Antes }^{\dagger} \\
\mathbf{N}=127\end{array}$} & \multirow{2}{*}{$\begin{array}{c}\text { Atual }^{\ddagger} \\
\mathbf{N}=127\end{array}$} & \multirow{2}{*}{ Valor $\boldsymbol{p}^{ \pm}$} \\
\hline & & & & \\
\hline \multirow[b]{2}{*}{ IQV Geral ${ }^{* *}$} & Satisfação & $26,7(2)$ & $25,3(2,3)$ & $<0,01$ \\
\hline & $\begin{array}{l}\text { Importân- } \\
\text { cia }\end{array}$ & $27,0(1,9)$ & $26,5(2,4)$ & 0,07 \\
\hline \multirow{2}{*}{$\begin{array}{l}\text { IQV Saúde/ } \\
\text { Funcionamen- } \\
\text { to*** }\end{array}$} & Satisfação & $26,8(2,4)$ & $24,1(3,4)$ & $<0,01$ \\
\hline & $\begin{array}{l}\text { Importân- } \\
\text { cia }\end{array}$ & $27,0(2,5)$ & $25,9(3,7)$ & $<0,01$ \\
\hline \multirow[b]{2}{*}{ IQV Família ${ }^{* * * *}$} & Satisfação & $28,1(2,4)$ & $28,1(2,4)$ & 0,986 \\
\hline & $\begin{array}{l}\text { Importân- } \\
\text { cia }\end{array}$ & $28,3(2,4)$ & $28,8(1,9)$ & 0,05 \\
\hline \multirow{2}{*}{$\begin{array}{l}\text { IQV Socioeco- } \\
\text { nômico }\end{array}$} & Satisfação & $26,8(2,9)$ & $26,1(3,0)$ & 0,078 \\
\hline & $\begin{array}{l}\text { Importân- } \\
\text { cia }\end{array}$ & $27,3(2,5)$ & $26,8(2,7)$ & 0,14 \\
\hline \multirow{2}{*}{$\begin{array}{l}\text { IQV Psi- } \\
\text { cológico/Es- } \\
\text { piritual }^{* * * * * * *}\end{array}$} & Satisfação & $27,5(2,9)$ & $27,0(3,0)$ & 0,196 \\
\hline & $\begin{array}{l}\text { Importân- } \\
\text { cia }\end{array}$ & $28,1(2,7)$ & $28,0(2,7)$ & 0,8 \\
\hline
\end{tabular}

† Média e desvio padrão antes do diagnóstico DMG; ${ }^{\ddagger}$ Média e desvio padrão após o diagnóstico de $\mathrm{DMG} ;{ }^{ \pm}$Valor de $p$ calculado pelo Teste $t$ Student; " Diabetes mellitus Gestacional; "* Índice de qualidade de vida geral; ${ }^{* * *}$ Índice de qualidade de vida sobre a saúde e funcionamento; ${ }^{* * * *}$ Índice de qualidade de vida familiar; ${ }^{* * * * * *}$ Índice de qualidade de vida socioeconômico; ${ }^{* * * * * *}$ Índice de qualidade de vida psicológico e espiritual.

\section{DISCUSSÃO}

Normalmente a gestação é um periodo que eleva o grau de preocupação da mulher. Essas preocupações geralmente giram em torno dos diferentes momentos da gravidez nas suas vidas; das questões financeiras que a gestação e o bebê acarretarão; da relação com o marido, que poderá ficar afetada em virtude do ciúme ou alteração na sexualidade que a gestação desencadeia; do medo de gerar um bebê com problemas de saúde ou malformado; e do medo em relação ao momento do parto
(PICCININI, 2012). Estudo relata que essas preocupações acentuam-se em gestantes diabéticas, devido à incerteza do prognóstico e andamento da gestação (MUNIM et al., 2012).

Parsons et al. (2014) afirmam a dificuldade de achar estudos específicos sobre as evidências dos efeitos metabólicos da DMG e qualidade de vida, salientando a importância de pesquisas que tragam à tona esses efeitos.

Nesse contexto, verifica-se o grau de satisfação das gestantes e, ao comparar os resultados desta satisfação antes e após o diagnóstico de DMG, há referência na queda do escore total de QV. Essa queda é reflexo sobre o IQV saúde/funcionamento. Estudos demonstram que o ato de modificar a alimentação, o uso de insulina ou hipoglicemiantes orais são determinantes para a insatisfação do paciente diabético, pois além de serem hábitos culturais, muitas vezes parte-se do pressuposto que a dieta balanceada é obrigatoriamente entendida como um regime e que difere essa pessoa das demais perante o seu convívio social. $\mathrm{O}$ ato de entender a doença e aceitá-la não é uma tarefa fácil a ser cumprida, bem como passa por inúmeros impasses ao longo do tratamento, que podem resultar em malefícios para o binômio mãe-filho. Munim et al. (2012), em seu estudo onde foram levantados os principais sentimentos vivenciados pelas gestantes diabéticas, relatam que durante a entrevista de sua amostra observou-se que as gestantes encontram dificuldades em dar continuidade ao tratamento devido a uma dieta restritiva. Esses mesmos autores também observaram que as gestantes, além de estarem preocupadas com a gestação em si, observam ainda sentimentos de medo e apreensão sobre a hora do parto.

Podemos notar que o IQV psicológico/espiritual, de forma geral, praticamente não apresentou alteração na avaliação das gestantes entrevistadas (a pontuação do escore geral é de 28), apesar de outras pesquisas trazerem que o período gestacional pode diminuir o funcionamento físico, vitalidade e aspectos sociais alterando o funcionamento psicológico e espiritual da mulher. E, ainda, esses aspectos poderiam reduzir o bem-estar e qualidade de vida das gestantes (TENTAIS et al., 2011). Além disso, outros autores afirmam que o apelo religioso auxilia no enfrentamento da gestação de alto risco, pois a "fé" e a "esperança" se fazem presentes, e são imprescindíveis para 
o controle de seu estado emocional (REZENDE, 2012). Na pesquisa desenvolvida por Parsons et al. (2014), é identificado que vários estudos abordam sentimentos de medo, apreensão vivenciados pelas gestantes diabéticas e preocupação com o seu filho.

Na pesquisa não houve alteração do IQV família, sendo que nesse quesito várias pesquisas apontam que a constituição de uma familia é algo muito esperado e ocasiona várias expectativas para a gestante (MARTINS et al., 2015). A falta de conhecimento e as crenças existentes sobre Diabetes mellitus Gestacional afetam o entendimento da gestante sobre as consequências da doença sobre o binômio mãe-filho, bem como podem impacatar sobre a gestação em si. Outro ponto importante relaciona-se com o alicerce indispensável que o convívio familiar traz para a gestante, pois o suporte familiar é fundamental, visto que, além do período gestacional há a presença do Diabetes mellitus Gestacional, necessitando de um processo educativo de todos os envolvidos no cuidado, guiado por valores e crenças do próprio paciente e de seu núcleo familiar acerca da doença (SCHMALFUSS et al., 2014).

Ressalta-se, dessa forma, que a organização familiar influencia fortemente o comportamento de saúde de seus membros e que o estado de saúde de cada indivíduo também influencia o modo como a unidade familiar funciona. Assim, infere-se que a família é uma instituição central que pode ajudar ou não a pessoa diabética a manejar a doença e alcançar as metas do seu tratamento (SCHMALFUSS et al., 2014).

O IQV socioeconômico também não apresentou valores significativos de impacto sobre os resultados gerais. Rezende (2012) pesquisou a Qualidade de Vida em gestantes de alto risco, conseguiu averiguar que a situação socioeconômica está relacionada às condições habitacionais, de higiene e alimentares e o resultado que ela obteve não demonstrou interferência sobre a QV, pois suas entrevistadas relacionaram o ambiente familiar como grande percursor para déficit em $\mathrm{QV}$, devido à ausência de um relacionamento conjugal estavél, onde a mãe solteria não possui um cônjuge para dividir as despesas e sustento da família, diminuindo sua qualidade de vida.

Os mesmos índices de QV foram novamente analisados através do grau de importância da QV para a gestante e apresentaram queda nos escores de IQV geral an- tes e após o diagnóstico, de 27,0 (DP: 1,9) para 26,5 (DP: 2,4). Além do IQV saúde/funcionamento de 27,0 (DP: 2,5) para 25,9 (DP: 3,7), e houve um terceiro escore alterado e de relevância que é o IQV socioeconômico, onde antes do diagnóstico era de 27,3 (DP: 2,5) e após o diagnóstico é de 26,8 (DP: 2,7). Meneguin e Xavier (2013) obtiveram, em seu estudo, queda nesse mesmo índice de IQV, onde sua casuísta equipara essa queda devido à gravidez não planejada e ao desemprego. No presente estudo não foi abordado o motivo da insatisfação dos domínios acima, apresentando uma fragilidade nos resultados comparativos.

Com certeza avaliar índice de qualidade de vida não é uma tarefa fácil, pois trata-se de um dado subjetivo. No entanto, nesse estudo quantificamos esse escore para obter melhor visualização desse índice. Meneguin e Xavier (2013) salientam que apesar de seu estudo com gestantes cardiopatas não ter apresentado queda geral da QV das entrevistadas, índices isolados possuíam tendência à queda, devido ao estado socioeconômico da gestante e a maternidade ser considerada um processo de inúmeras transformações tornando-se complexo para a mulher. Associando esse cenário à uma doença, há gradativamente o aumento das preocupações e anseios. No entanto, existe um grau de dificuldade em achar estudos específicos sobre as evidências dos efeitos metabólicos da DMG, apesar de haver grande importância de pesquisas desse gênero (PARSONS et al., 2014).

Contudo, algumas limitações deste estudo devem ser reconhecidas. A escassez de estudos sobre a qualidade de vida em pacientes gestantes dificultou a comparação dos resultados com a utilização da ferramenta de Ferrans e Powers - Quality of Life Index - Diabetes Version III; nesse estudo, não foram abordados os possíveis motivos da insafistação que conduz à queda do escore de qualidade de vida; e o instrumento utilizado não possui um parâmetro específico para analisarmos o grau de qualidade de vida da casuísta. Mas, por outro lado, mostrou que futuras pesquisas certamente devem ser conduzidas nesta área. 


\section{CONCLUSÃO}

A intenção deste estudo foi identificar a influência do diagnóstico de Diabetes mellitus Gestacional sobre a qualidade de vida da gestante e traçar o perfil das gestantes portadoras da doença. Para tanto, realizou-se um estudo comparativo entre os resultados obtidos antes e após o diagnóstico de Diabetes mellitus Gestacional. Sendo assim, considera-se que os objetivos foram alcançados e respondem aos propósitos da Linha de Pesquisa Saúde e Meio Ambiente, pois essa doença está intrinsecamente relacionada com o meio social e ambiental da gestante.

Os achados desta pesquisa permitem afirmar que o diagnóstico de Diabetes mellitus Gestacional altera a qualidade de vida da gestante; no entanto, são dados subjetivos que fornecem a perspectiva da casuística estudada.

Observou-se queda no IQV geral, sobre reflexo de queda do IQV saúde/funcionamento. Esses dados são específicos, porém não foram pesquisadas as circunstâncias que favorecem essa queda.

Esse estudo demonstra a importância de levar em consideração a vivência das gestantes diabéticas, bem como essas vivências assumem papéis importantes para o tratamento e andamento da gestação. No entanto, devido à escassez de outras pesquisas nesse âmbito, há dificuldades em relacionar os dados obtidos com as referências existentes. Sendo assim, torna-se essencial haver continuidade de estudos nesses patamares de conhecimento e que possibilitem a integração com o tratamento e cuidados gerais da gestante diabética.

\section{REFERÊNCIAS}

ABI-ABIB, R. C.; CABIZUCZ, C.; CARNEIRO, J. R. I.; BRAGA, F. O.; COBAS, R. A.; GOMES, M. B.; JESÚS, G. R.; MIRANDA, F. R. D. Diabetes na gestação. Rev HUPE, Rio de Janeiro, v. 13, n. 3, p. 40-47, jul. 2014.

CARNEIRO, M. J.; EUSÉBIO, C. Segmentation of the tourism market using the impact of tourism on quality of life. TMStudies, v. 7, p. 91-100, 2011.
FAGUNDES, A. A.; BARROS, D. C.; DUAR, H. A.; SARDINHA, L. M. V.; PEREIRA, M. M.; LEÃO, M. M. Vigilância alimentar e nutricional - SISVAN: orientações básicas para a coleta, processamento, análise de dados e informação em serviços de saúde. Brasília: Ministério da Saúde, 2004. 122p. (Série A. Normas e Manuais Técni$\cos )$.

FERREIRA, R. L. Doenças, germes e curas: a história da medicina no Brasil Colonial. Rev Eletr Cad Hist, v. 8, n. 1, jul. 2013.

MARTINS, Q. P. M.; FERREIRA, G. S. M.; ARAGÃO, A. E.; GOMES, F. M. A.; ARAÚJO, L. M.; FERREIRA, F. I. S. Conhecimentos de gestantes no pré-natal: evidências para o cuidado de enfermagem. SANARE, Sobral, v. 14, n. 2, p. 65-71, jul./dez. 2015.

MENEGUIN, S.; XAVIER, C. de L. Qualidade de vida em gestantes cardiopatas. Texto Contexto Enferm., Florianópolis, v. 22, n. 3, p. 811-818, jul./set. 2013.

MINISTÉRIO DA SAÚDE. Conselho Nacional da Saúde. Resolução no 466, de 12 de dezembro de 2012. Trata de pesquisas em seres humanos e atualiza a resolução 196. Brasília, DF, 14 jun. 2013. Disponível em: < http:// conselho.saude.gov.br/ultimas_noticias/2013/06_ jun_14_publicada_resolucao.html $>$. Acesso em: 12 nov. 2015.

MUNIM, R. A. de S. et al. Sentimentos vivenciados pelas gestantes portadoras de Diabetes Mellitus. Rev Biotecnol. Ciênc., Anápolis, v. 2, n. 1, p. 62-82, dez. 2012.

OLIVEIRA, A. R.; CARVALO, T. A. Consumo e descarte: formas de agenciamento da subjetividade humana na contemporaneidade. Cad Zg ymunt Bauman, Paraíba, v. 4, n. 7, p. 35-49, 2014.

PARSONS, J.; ISMAIL, K.; AMIEL, K.; FORBES, A. Perceptions among women with gestational diabetes. Qual Health Res, v. 24, n. 4, p. 575-585, abr. 2014.

PICCININI, C. A. Percepções e sentimentos de gestantes sobre o Pré-natal. Psic. Teor. e Pesq., Brasília, v. 28, n. 1, p. 27-33, jan./mar. 2012. 
RAMOS, A. M. B. Esperança no contexto da gravidez de risco: contributo para o estudo da esperança no processo gravídico de risco médico, psicológico e social. 2014. 35f. Dissertação (Mestrado em Psicologia) - Instituto Superior Miguel Torga, Coimbra.

REZENDE, C. L. Qualidade de vida das gestantes de alto risco em centro de atendimento à mulher do município de Dourados, MS. 2012. 128f. Dissertação (Mestrado em Psicologia) - Universidade Católica Dom Bosco, Campo Grande, MS, 2012.

SCHMALFUSS, J. M.; PRATES, L. A.; AZEVEDO, M.; SCHNEIDER, V. Diabetes melito gestacional e as implicações para o cuidado de enfermagem no pré-natal. Cogitare Enferm., Chapecó, v. 19, n. 4, p. 815-822, out./dez. 2014.

SILVEIRA, C.; FERREIRA, M. Auto-conceito da grávida: factores associados. Millenium, v. 40, p. 53-67, 2011.

STATEMENTS, P. Standards of medical care in diabetes. Diabetes Care, v. 35, n. 1, p. 11-63, jan. 2012.

TENTAIS, I.; FIGUEIREDO, B.; MOTA, J.; CONDE, A. Atividade física, qualidade de vida e depressão durante a gravidez. Cad Saúde Pública, Rio de Janeiro, v. 27, n. 2, p. 219-228, fev. 2011.

Recebido em: 18 outubro 2015 Revisado em: 23 novembro 2015

Aceito em: 25 novembro 2015 